\title{
Recruiting microbial bodyguards
}

Nat. Biotechnol. http://doi.org/cvth (2018).

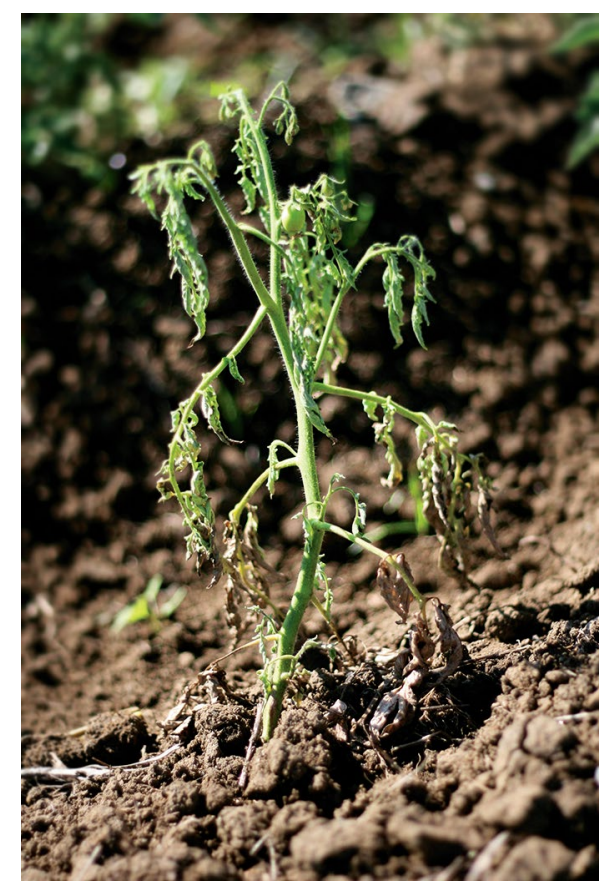

Credit: AGRIOLOGY / ALAMY STOCK PHOTO

Soil is more than decaying organic matter and some rocks. A teaspoon of a healthy topsoil layer probably contains billions of microorganisms encompassing thousands of species, many of them not yet described. The current unabating progress in sequencing power - enabling increasingly sensitive metagenomic studies - is helping to reveal the functional importance of the soil microbiome and its effect on plant development, nutrition and responses to environmental changes.

Writing in Nature Biotechnology, Jihyun Kim and colleagues demonstrate that in tomato, the microbiome composition is critical for resistance to Ralstonia solanacearum, a soil pathogen that colonizes the xylem and causes bacterial wilt. The team applied a metagenomics approach to compare the rhizosphere microorganisms of resistant and susceptible tomato varieties. Transplant experiments showed that the soil by itself confers some level of resistance to the susceptible genotype. Finally, the authors isolate and characterize a novel flavobacterium that can partially suppress bacterial wilt symptoms when added to soil.

This study reveals that plants recruit beneficial microorganisms to help them fight against pathogens or more generally to tolerate non-optimal conditions. It is easy to conceive potential strategies to protect crops from diseases in the future, for example by directly inoculating soils or seeds, or by modifying genomes to exudate attractive chemicals, in order to recruit the right kind of beneficial bacteria. Crop diseases are responsible for huge economic losses and threaten food security worldwide, and every solution is welcome.

\section{Guillaume Tena}

Published online: 2 November 2018 https://doi.org/10.1038/s41477-018-0308-5 